\title{
STIS observations of FLIERs in NGC 7662^
}

\author{
M. Perinotto ${ }^{1}$, P. Patriarchi ${ }^{2}$, B. Balick ${ }^{3}$, and R. L. M. Corradi ${ }^{4}$ \\ 1 Dip. di Astronomia, Universita' di Firenze, Largo E. Fermi 5, 50125 Firenze, Italy \\ e-mail: mariop@arcetri.astro.it \\ 2 IRA/CNR, Largo E. Fermi 5, 50125 Firenze, Italy \\ 3 Astronomy Dept., FM-20, University of Washington, Seattle - WA 98195, USA \\ ${ }^{4}$ Isaac Newton Group of Telescopes, Ap. de Correos 321, 38700 Sta. Cruz de la Palma, Canary Islands, Spain
}

Received 10 November 2003 / Accepted 15 March 2004

\begin{abstract}
We observed the planetary nebula NGC 7662 with STIS on board the HST to investigate the physical conditions in the microstructures within the nebula at high spatial resolution and to closely examine the physical conditions in FLIERs. We aim to reveal spectroscopic differences in small scale structures moving at about the same velocity as the surrounding nebular gases, called SLOWERs. Shocks are expected to play a role in FLIERs but not in SLOWERs. The physical conditions in the two types of microstructures have been determined better than ever. In both types of substructures, the electron density shows a moderate increase inward, while the electron temperature becomes a little lower. The ionization increases from the central parts of the FLIERs to their edges. No particular increase of the line emission typical of shock excitation, such as the [SII] $6725 \AA$ doublet, is seen at the interface between the ambient gas and the microcondensations. Thus, we observe no evidence for shock effects in the FLIERs. We estimate that the thickness of the post-shock layer is too small to be detected, in spite of the brightness of the nebula. For the same reason the determinations of abundances within the FLIERs done assuming the contribution of photoionization followed by ion-electron collision excitation to be dominant in producing the observed emission lines, might be considered to be acceptable. The geometrical complexity of microstructures composed of sub and subsub-structures coupled with the need to take into account charge-exchange reaction effects, prevent us from drawing a firm conclusion on the nitrogen overabundance suggested in the literature for the FLIERs relative to the surrouding ambient gas. We however argue that our HST observations do not support this enhancement.
\end{abstract}

Key words. ISM: Planetary nebulae: individual: NGC 7662 - stars: AGB and post-AGB

\section{Introduction}

The name FLIERs (Fast Low Ionization Emission Regions) was introduced by Balick (1987) to indicate high-velocity microstructures with arcsecond size that are prominent in lines of low ionization ions, and that are seen on opposite sides of the central star along the major axis of elliptical planetary nebulae (PNe). Their high outward velocity relative to the surrounding gases together with their occurrence in pairs, located nearly symmetrically with respect to the central star, has attracted the interest of many researchers. Consequently much important observational work has been done from the ground.

Two classes of model have been proposed: i) clumps of gas resulting from hydrodynamical interaction of colliding flows; and ii) high speed "bullets" ejected by the central object. The first proposes hydrodynamical focusing of an initially isotropic "fast" stellar wind (Balick et al. 1987, hereafter "BPI"; see for

^ Based on observations made with the NASA/ESA Hubble Space Telescope, obtained at the Space Telescope Science Institute, which is operated by the Association of Universities for Research in Astronomy, Inc., under NASA contract NAS 5-26555. These observations are associated with program GO-8128. properties of winds: Patriarchi \& Perinotto 1991) by oblique shocks associated with the non-spherical, dense low-velocity wind emitted in the AGB phase. This would produce an enhanced material density in the outer parts of the main body of the elliptical nebula along its major axis (Balick 1987; Frank et al. 1996). This collimation process was first criticized by Soker (1990) who proposed instead a bullet origin of FLIERs, and then also by Mellema (1996). In the bullet scenario two blobs are symmetrically ejected at high speed from the central source. The ejection may be due to the interaction of the progenitor star with a binary companion, which is either stellar in size (Livio 1982; Soker \& Livio 1994; Livio \& Pringle 1996) or substellar (Soker 1992; Harpaz \& Soker 1994). After a common envelope phase, the companion (preferably a brown dwarf or super-Jupiter type planet) would collide with the progenitor of the PN causing the formation of a disk from which two opposite jets might be blown away. In the bullet hypothesis one should however explain how a blob of gas would move through an ambient medium of similar density (as found by Balick et al. 1994; hereafter Ba94) to the edge of the nebula without being destroyed. 
Several authors have discussed specific aspects of the topic. Dopita (1997) proposed a hydrodynamical model of the FLIER taking into account the role of magnetic fields. Mellema et al. (1998) considered the role of the stellar radiation field which may erode the blob by photo-evaporation; Redman \& Dyson (1999) analyzed the mass loading processes caused by the fast wind, while Pascoli (1997) discussed the role of magnetic fields initiated by turbulent or regular motions within the degenerate core of the AGB star, persisting across the ejected material and thus contributing to its shaping.

Garcia-Segura (1997) calls for a 3D magnetic collimation process to account for the formation of FLIER-like microstructures.

According to Balick et al. (1998) none of these models is able to satisfactorily reproduce all the various aspects of the ground based imaging and spectroscopical observations, while, following Soker \& Regev (1998), the "bullet" hypothesis is still viable (see also the interesting paper by Steffen et al. 2001).

Recently, many PNe were observed with the WFPC2 on HST, including practically all those known to contain FLIERs (cf. Balick et al. 1998; Valdettaro et al. 2001). To ascertain the real nature of FLIERs it is clearly very important to perform spectroscopic investigation at the high spatial resolution provided by HST.

In this paper we present results of a spectroscopic study done with STIS on HST of features considered to be FLIERs, and of low-velocity knots (non-FLIERs) belonging to the same planetary nebula: NGC 7662. The work has two aims: first, to investigate the physical properties of FLIERs at the highest spatial resolution; second, to search for differences, if any, between FLIERs and non-FLIERs (also named SLOWERs: Slow Moving Low Ionization Emitting Regions, Perinotto 2000). In Sect. 2, the observations and data reduction are presented. Section 3 illustrates the large-scale trends of the physical parameters of the nebula along the slit. Section 4 specifically discusses and compares FLIERs and SLOWERs and examines some of the more plausible interpretations of the data. A summary and the conclusions are in Sect. 5.

\section{Observations and data reduction}

Our observational goals were to study the ionization structure, physical conditions (i.e., density and temperature), abundances, and kinematic structure of selected low-ionization knots. Using BPI's ground-based Echelle observations of NGC 7662 as a guide, we observed in two slit positions, each passing near the central star so that we could verify the exact location of the slit. The slit placements are shown in Fig. 1 along with the identifications of the global structures of the PN, namely the "central bubble", "rim" and "shell".

The grating tilts and integration times were selected to cover key parts of the spectrum.

Two gratings (G430L and $\mathrm{G} 750 \mathrm{M}$ ) were used in combination with the $52^{\prime \prime} \times 0.5$ slit to measure line fluxes. Grating G750M was also used with the $52^{\prime \prime} \times 00^{\prime} 1$ slit in order to search for variations of the Doppler shift in the gas that connects FLIERs and SLOWERS to the nebular core.
The grating G750M in two tilts corresponding to the central wavelengths $\lambda 6581$ and $\lambda 5734 \AA$ covered the interval $\lambda 6295$ to $\lambda 6867 \AA$ and $\lambda 5448$ to $\lambda 6020 \AA$, respectively, with reciprocal dispersion of $0.56 \AA \mathrm{pix}^{-1}$. The grating G430L covered the spectral range 2900 to $5700 \AA$ with reciprocal dispersion of $2.73 \AA \mathrm{pix}^{-1}$. See Table 1 for the integrations times and other details. The search for kinematic variations was not successful owing to the poor spectral resolution of the $6750 \mathrm{M}$ grating and the lack of a strong signal through the narrow slit.

Position 1 (July 2000, called POS 1) passes through the main large scale components of NGC 7662, namely the northern and southern regions of the central bubble, rim, and shell, as well as the microstructures that we call FLIER-a (pixels 131-135) and FLIER-b (pixels 144-156) south of the nucleus. They appear projected on the southern side of the shell. FLIER-a and FLIER-b have been termed in previous papers "inner sinewy FLIER" and "outer sinewy FLIER", respectively. BPI found them to have a velocity of $\sim 70 \mathrm{~km} \mathrm{~s}^{-1}$ with respect to the overall expansion velocity of the entire nebula $\left(\sim 25 \mathrm{~km} \mathrm{~s}^{-1}\right)$.

Position 2 (POS 2) also goes though the main large-scale components of NGC 7662, and covers completely a SLOWER near the NE edge of the nebula and partially a second SLOWER on the opposite side of the nebula.

In all cases we used the standard STIS pipeline calibration procedure which corrects for bad pixels, applies corrections for nonuniform CCD illumination and geometrical distortions, and converts detected counts to energy flux per pixel. An onboard binning of 2 pixels in the spectral direction and 4 pixels in the spatial direction was applied to all data obtained through the wider slit in order to improve the signal to noise of the data with only a modest loss in resolution. Each binned pixel resulting of the wide slit spectra thus projects on the sky onto $0.1 \times 0.2$ arcsec, while the (unbinned) pixel of the narrow slit spectra is of $0.05 \times 0.05 \mathrm{arcsec}$. Cosmic rays were identified and removed when averaging repeated observations.

\section{Results: Large-scale trends}

Figure 1 is an overlay of HST images in the lines of $\mathrm{N}^{+}$ (red), $\mathrm{O}^{++}$(green), and $\mathrm{He}^{++}$(blue) from Balick et al. (1998). Figure 1 shows that both slits cross characteristic portions of the central bubble (which is probably invisible), the rims that surround the bubble, and the shell of smoothly distributed gas (other than the FLIERs and SLOWERs) between the rims and the FLIERs and SLOWERs. Hence we can separately derive properties of the large-scale structure (this section) and the small (next section).

\section{1. $\mathrm{H}$ and $\mathrm{He}$ lines and reddening across the nebula}

The hydrogen Balmer lines have excellent $\mathrm{S} / \mathrm{N}$ throughout NGC 7662 (an example in the regions of the FLIERs and SLOWERs can be found in the lower right panels of Figs. 2 and 3). There are no signs of differential reddening at any spatial scales, including those corresponding to the FLIERs and SLOWERs. Similar results are obtained by considering the 


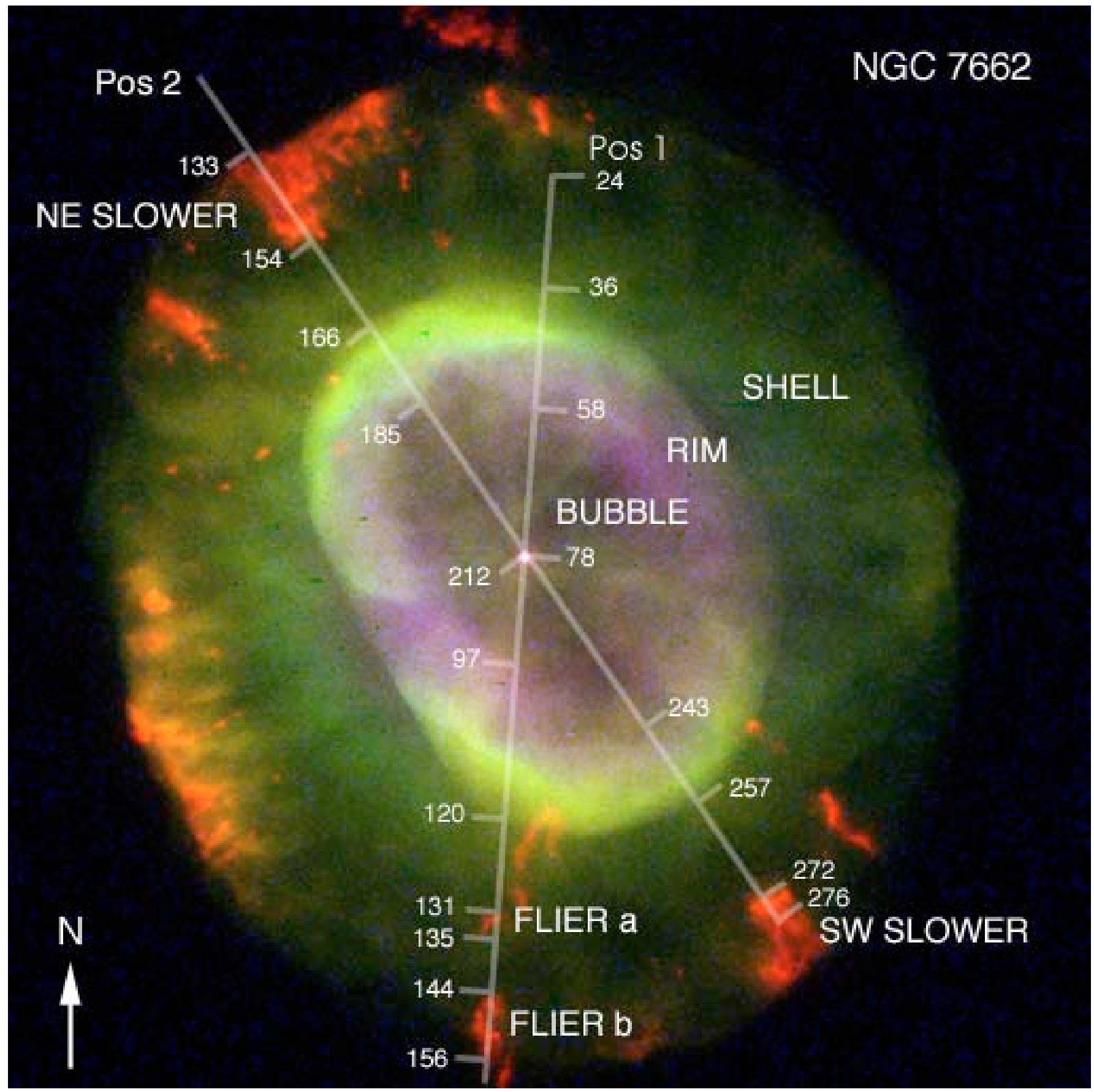

Fig. 1. Composite HST WFPC2 images of NGC 7662. Red is [NII] radiation $\lambda 6583 \AA$, green is [OIII] $\lambda 5007 \AA$, blue is HeII $\lambda 4686 \AA$. Locations of the STIS slit are indicated for the two observed positions POS 1 and POS 2. The white dot indicated the central star. The various macro-features - bubble, rim and shell - as well as the microstructures - FLIER-a, FLIER-b, SLOWER - are marked. Pixel numbers from the wide slit spectra are also marked; one pixel corresponds to 0.2 arcsec. The center of the slit in POS 1 and in POS 2 is around pixel 150 . (This figure is available in color in electronic form at http://www.edpsciences.org)

He I recombination lines, in spite of their lower S/N. The spike in the ratio in Fig. 3 is a one-pixel anomaly.

The $\mathrm{H} \alpha / \mathrm{H} \beta, \mathrm{H} \gamma / \mathrm{H} \beta$ and $\mathrm{H} \delta / \mathrm{H} \beta$ line ratios are constant to a high degree of accuracy across the whole nebula. Their average values are 3.3, 0.42, 0.24 in POS 1 and 3.3, 0.41, 0.23 in POS 2, respectively.

In the same zones, the HeI 4471/5876 and HeI 6678/5876 line ratios take the values 0.361 and 0.280 (POS 1), and 0.357 and 0.278 (POS 2), while the HeII 3201/4686 A, HeII 5411/4686 and HeII 4540/4686 ratios amount to $0.35,0.085$ and 0.021 , respectively, in POS 1 , and $0.36,0.078$ and 0.020 in POS 2. All these He line ratios are also remarkably constant throughout the whole nebula, as illustrated in later figures.

Using our Balmer line ratios and the Cardelli et al. (1989) extinction law we derive a reddening constant $c_{\beta}=0.2$. This agrees with many other values found in the literature except for Hyung \& Aller (1997) who adopted $c(\mathrm{H} \beta)=0.1$. 
Table 1. Observational parameters.

\begin{tabular}{|c|c|c|c|c|c|c|}
\hline & & & & $\begin{array}{l}\text { G430L } \\
(\lambda 4300)\end{array}$ & $\begin{array}{l}\text { G750M } \\
(\lambda 6581)\end{array}$ & $\begin{array}{l}\text { G750M } \\
(\lambda 5734)\end{array}$ \\
\hline Aperture & RA & Dec & PA & \multicolumn{3}{|c|}{ Exposure time (s) } \\
\hline Position 1 & 232553.67 & 423150.8 & $193^{\circ}$ & & & \\
\hline $52 \times 0.1$ & & & & & 2108 & \\
\hline $52 \times 0.5$ & & & & 5802 & 2733 & 11079 \\
\hline Position 2 & 232553.96 & 42325.3 & $231^{\circ}$ & & & \\
\hline $52 \times 0.1$ & & & & & 2110 & \\
\hline $52 \times 0.5$ & & & & 5802 & 2733 & 11081 \\
\hline
\end{tabular}

The measured fluxes of all observed spectral lines have been corrected with $c(\mathrm{H} \beta)=0.2$ and normalized to $\mathrm{H} \beta=100$.

The constancy of the above line ratios rules out dense concentrations of dust and the possibility to have revealed strong shocks in which locally high densities and temperatures might cause excited levels of hydrogen to be populated by collisions out of the ground level, as opposed to the usual nebular level populations of excited levels by recombinations and cascades. This was predicted by Dopita (1997) for the case where the energy density of shocks is less than that of the local stellar radiation field.

\subsection{Ionization structure}

There are a variety of ionization states in NGC 7662, from low $\left(\mathrm{H}^{+}, \mathrm{He}^{\mathrm{o}}\right)$, moderate $\left(\mathrm{H}^{+}, \mathrm{He}^{+}\right)$, and high $\left(\mathrm{H}^{+}, \mathrm{He}^{++}\right)$. These regions as red, green, and blue portions of Fig. 1, respectively.

Using terminology defined earlier, the bubble is almost certainly invisible since it is heated by the stellar wind; its temperature $\left(>10^{6} \mathrm{~K}\right)$ is too high, and its emission measure is too low (density $n<10^{-3} \mathrm{~cm}^{-3}$ ) for optical emission to be detected above the surface brightness of other nebular regions. The rim seems to be a bright and relatively dense and photoionized transition region in which the ionization state of helium drops from high to moderate. The shell is a region of moderate ionization, bright in lines such as $\mathrm{H} \alpha$ and $\mathrm{O}^{++}$, except for the compact low ionization regions discussed in the next section.

For helium the line flux ratio of $\left(\mathrm{He}^{+}+\mathrm{He}^{++}\right) / \mathrm{H}^{+}$is constant even though $\mathrm{He}^{+} / \mathrm{He}^{++}$varies from the zone projected towards the bubble (likely the corresponding radiation comes from the foreground and background rim and shell, see next section) to the shell as said above. This indicates the constancy of the total abundance of $\mathrm{He} / \mathrm{H}$ across the nebula.

\subsection{Density and temperature}

Our ability to measure density is limited largely to regions where the [S II] lines are bright - i.e. the regions where $\mathrm{He}$ is entirely or partially neutral (which includes the FLIERs and SLOWERs but excludes the bubble and the adjacent rim). Fortunately we can also determine the electron temperatures in these regions from the [N II] line ratios.
The temperature $T_{\mathrm{e}}$ is easily measured in regions of the rim and shell where $\mathrm{O}^{++}$is abundant using [O III] line ratios. We find an electron temperature of $14000 \mathrm{~K}$ in the highly ionized portions of the rim. As the slit crosses through the rim (in projection) the $\mathrm{O}^{++}$temperature drops steadily to about $12000 \mathrm{~K}$ in the outside of the rim and the adjacent shell. This is expected in photoionization models (e.g., Alexander \& Balick 1997 , hereafter "AB97") since $\mathrm{O}^{++}$is an important coolant in zones of moderate ionization. Note that oxygen is largely neutral or singly ionized in regions of low ionization.

The densities of the rim and shell are difficult for us to measure since diagnostic lines such as [Cl III] are weak. The noisy [Cl III] data have been summed in the rims and yield a density of 5000-9000 $\mathrm{cm}^{-3}$ with large uncertainty. No density estimate is possible from [ $\mathrm{Cl} \mathrm{III}]$ in the shell.

Somewhat surprisingly the $[\mathrm{S} \mathrm{II}]$ lines reach a maximum in the rim, arguing that low-ionization ions are present there. The [S II] line ratio yields a density of $4000 \mathrm{~cm}^{-3}$ in this region where both slits, POS 1 and POS 2, cross it. For POS 1, between FLIER-a and FLIER-b, i.e. in the shell, the $\mathrm{S}^{+}$density amounts to $1300 \mathrm{~cm}^{-3}$. The same values are found in the shell beyond FLIER-b. For POS 2 a density closer to $1800 \mathrm{~cm}^{-3}$ in the shell outside of the SLOWER.

\section{Results: Trends in the FLIERs and SLOWERs}

Plots of the surface brightness of selected lines and line ratios in the regions around the FLIERs and the SLOWER are shown in Fig. 2 (POS 1; FLIERa and FLIERb) and Fig. 3 (POS 2; SLOWER) with an effective spatial resolution of $0.1^{\prime \prime} \times 0.2^{\prime \prime}$. As noted in the figure captions, some smoothing has been used to improve the signal-to-noise ratio where data are particularly weak. The results of smoothing are shown with vertical bars that span the pixels used in the flux averages. (This averaging was essential for estimating temperatures using the [N II] $\lambda 5755 \AA$ A line and densities from the [S II] lines in certain locations.) In order to illustrate the line brightness profiles of the strongest lines at the full HST spatial resolution, we also show in Fig. 4 the plots for the narrow slit spectra.

Figures 2-4 show clearly that FLIERs and SLOWERs have bright peaks - that is, high emission measures - in lines of low ionization, such as [O I] $\lambda 6300 \AA$, [O II] $] \lambda 3727 \AA$, 

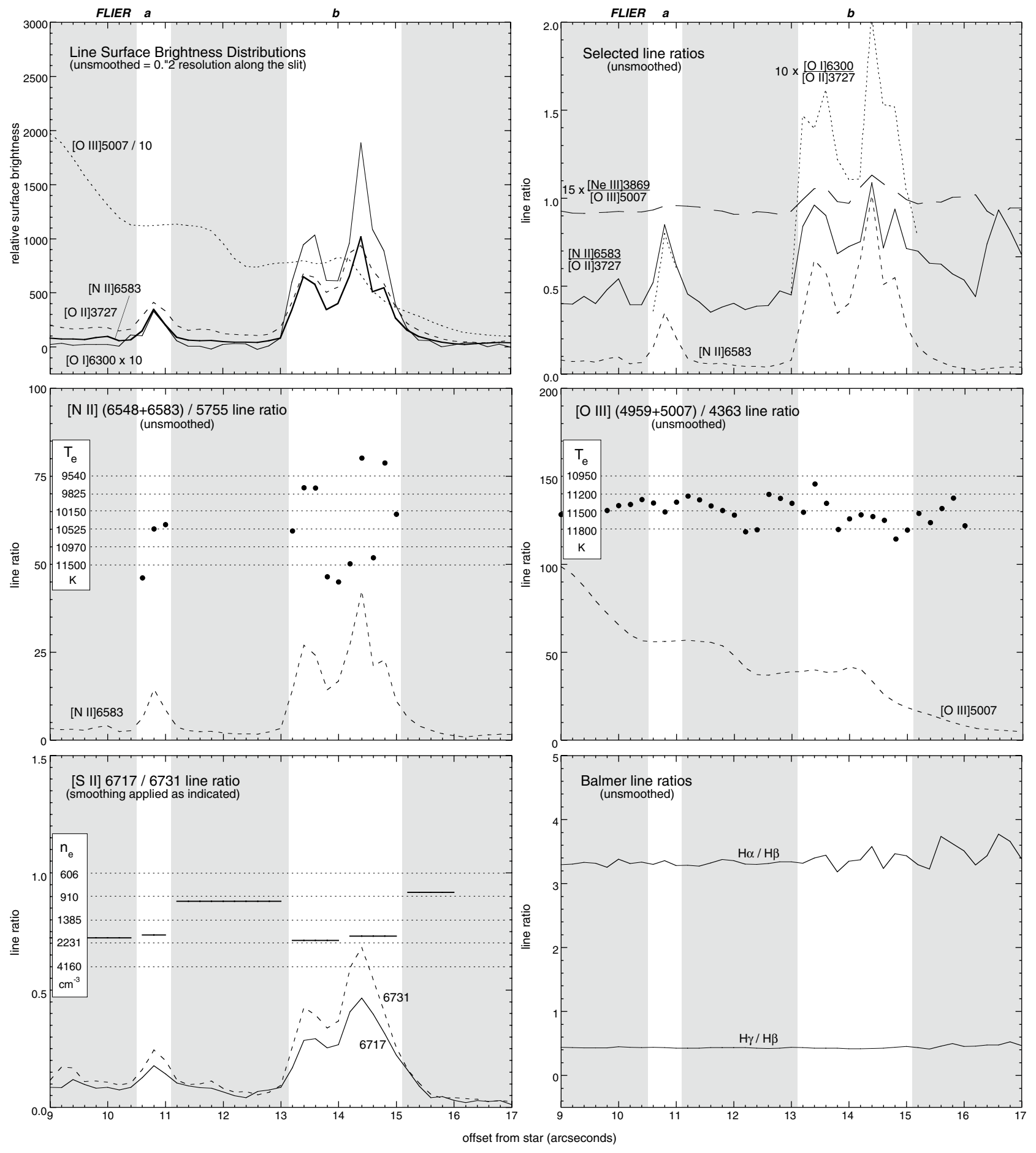

Fig. 2. Spectroscopic results for portions of POS 1 using the $0.1^{\prime \prime}$-wide slit and averaging the flux over $0.2^{\prime \prime}$ along the slit. FLIER-a is centered at $\sim 10.8^{\prime \prime}$ from the star, and FLIER-b is about $14^{\prime \prime}$ from the star. Plotted fluxes have been scaled as indicated for clarity. Some data have been averaged over more than one pixel for better $\mathrm{S} / \mathrm{N}$; these results are shown as horizontal bars that cover the zones that were averaged together.

and $[\mathrm{N} \mathrm{II}] \lambda 6583 \AA$. This, and the high density measured at these peaks (below), warns us that collisional de-excitation can be important for those lines with relatively low critical densities, such as the $[\mathrm{S} \mathrm{II}]$ and $[\mathrm{O} \mathrm{II}]$ lines. We expect to find that the peaks of these lines are somewhat suppressed relative to, say, [N II]. Indeed, this expectation is realized. Then our derived densities at the brightness peaks are probably lower limits; the true densities are likely $\sim 10^{4} \mathrm{~cm}^{-3}$. (An additional complication that affects the interpretation of line ratios is charge exchange. More about that later.)

\subsection{Ionization structure}

The narrow-slit, high-resolution plots in Fig. 4 show that the FLIER is not prominent in the lines of $\mathrm{H} \alpha, \mathrm{O}^{++}$or $\mathrm{Ne}^{++}$. The last two lines show only a marginal enhancement near 

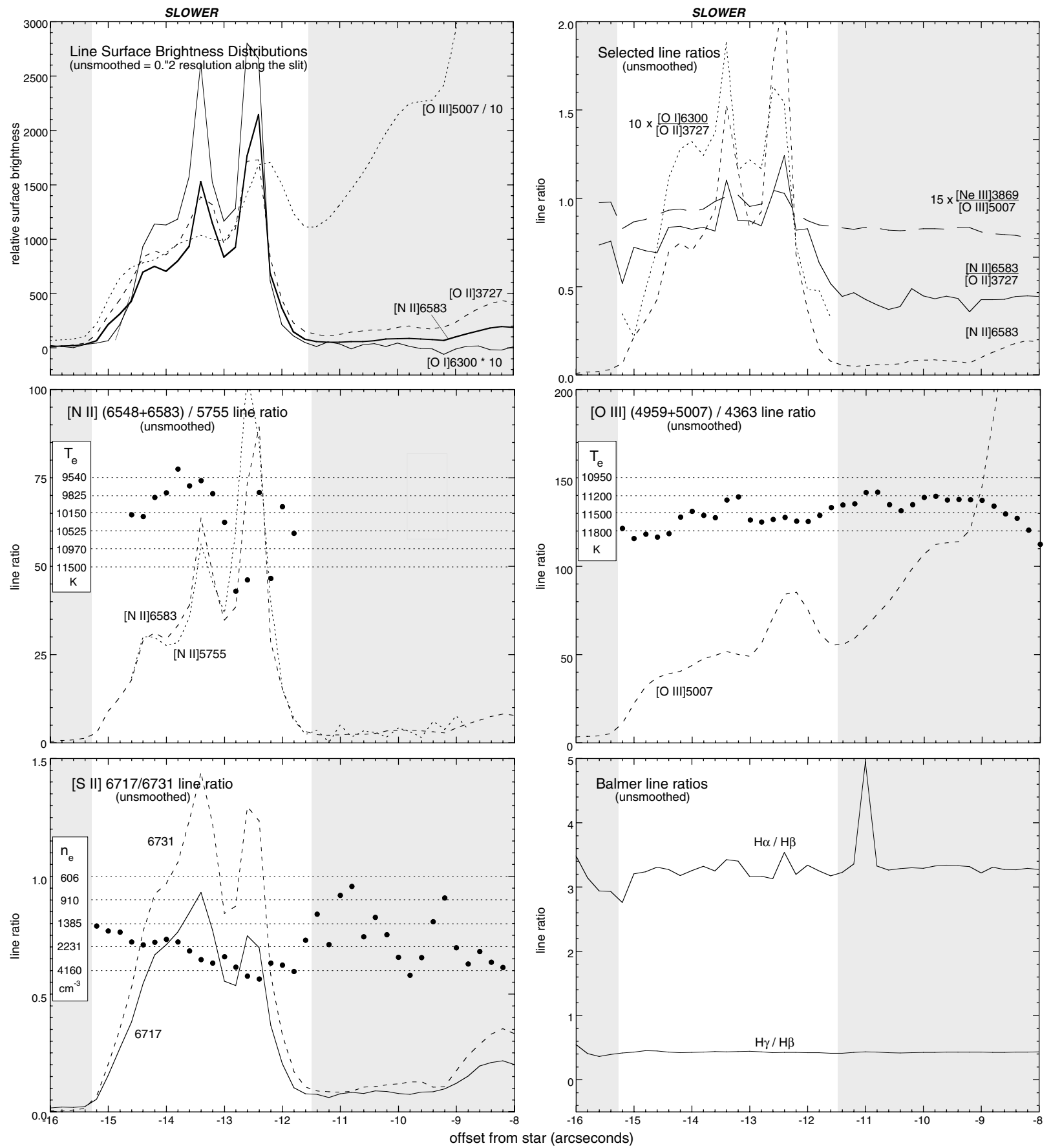

Fig. 3. The same as in Fig. 2, but for the NE SLOWER (around -13 arcsec) at POS 2. The central star is to the right.

the star-facing edge of the SLOWER. H $\alpha$ peaks on the leading (star-facing) edge of the SLOWER and then fades to the outer edge of the nebula. In contrast, the [O I] $\lambda 6300 \AA$ line rises dramatically in the low-ionization regions, as seen if Figs. 2 and 3. [O I] $\lambda 6300 \AA$ approaches $20 \%$ of [N II] $\lambda 6583 \AA$, which is unusually large. The $[\mathrm{O} \mathrm{I}]$ peak is marginally displaced on the far side of the star from the [N II] peak.

These trends point towards FLIERs and SLOWERs having partially neutral cores where a significant fraction of hydrogen is present. There is likely to be an ionization front interface between the neutral and ionized gas outside, possibly resulting in a strong thermal pressure gradient across the interface.
Near the partially neutral interior charge exchange reactions in which $\mathrm{H}^{\mathrm{o}}$ donates its electron to neutral oxygen and nitrogen can affect the ionization balance of all of these ionic species. In particular, $\mathrm{O}^{+}$will be depleted faster than $\mathrm{N}^{+}$(Osterbrock 1989), enhancing the [N II]/[O II] line ratio over the value expected in the absence of charge exchange.

\subsection{Density structure}

The lower left panels in Figs. 2 and 3 show the [SII] $\lambda 6717 / \lambda 6731$ line ratio profile. The relationship between the line ratios and densities $n_{\mathrm{e}}$ are indicated in the same panels. 

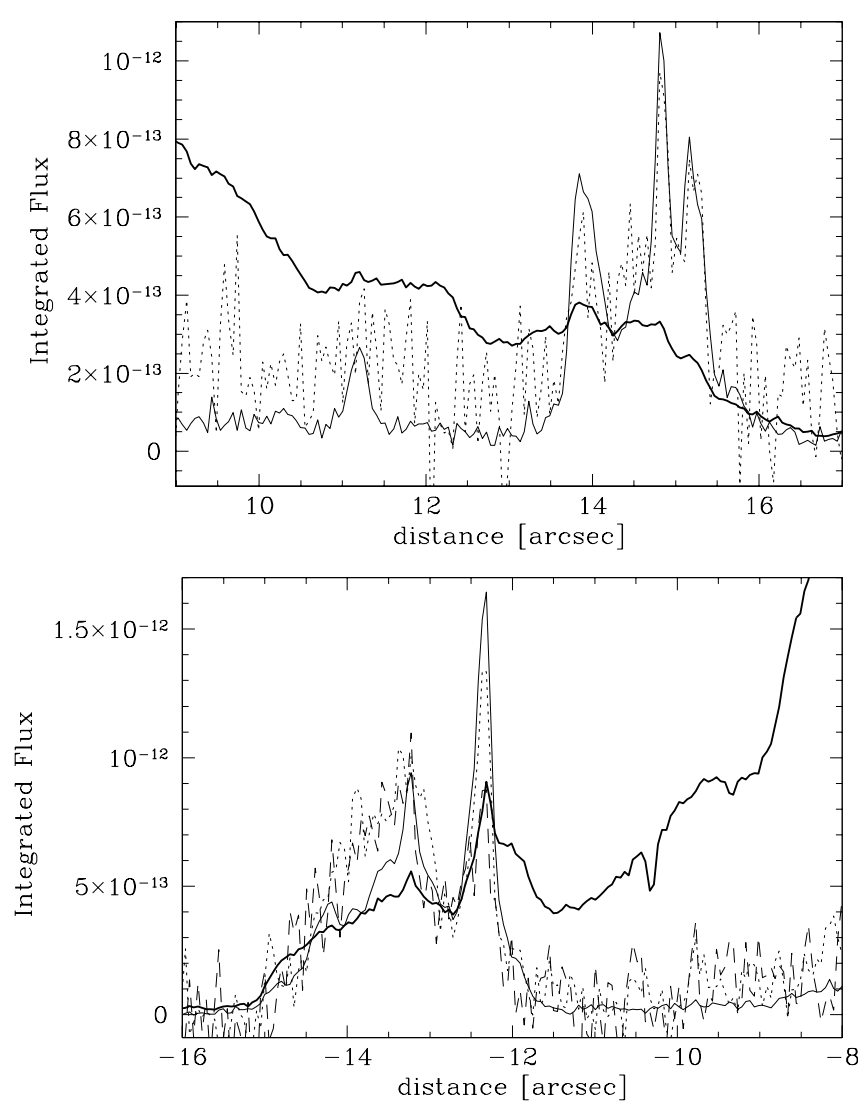

Fig. 4. Top: line brightness profiles from the narrow slit spectra (width $=0.05^{\prime \prime}$ ) at POS 1 . The central star is to the left. The portion of the nebula covering FLIER-b (at $11.3 \mathrm{arcsec}$ ) and FLIER-a (around $15 \operatorname{arcsec}$ ) is shown. Solid line is $\mathrm{H} \alpha$, the thin one is [NII]6583 $\AA$, and the dotted line is [SII]6731 $\AA$. Bottom: the same for POS 2, covering the NE SLOWER (around -13 arcsec from the central star). Line styles as above, with the addition of the dashed line for [SII]6717 $\AA$. Central star to the right.

(The individual [SII] lines are plotted for reference and to show the quality of the data.) As noted above, the density derived from the [S II] line ratio is likely to be an underestimate.

We believe that there is consistent evidence that the densities near the peaks of the $\mathrm{S}^{+}$zones of the FLIERs and SLOWERs are at least twice as large as in their surroundings. The density contrast could be larger since the shown data have been smoothed and because quenching suppresses emission from the densest regions. The actual numbers are of $2200 \mathrm{~cm}^{-3}$ for the density in FLIER-a and FLIER-b, and $3000 \mathrm{~cm}^{-3}$ in the SLOWER.

\subsection{Temperature structure}

The panels in the middle rows of Figs. 2 and 3 present line profile ratios that are sensitive to the electron temperature. The corresponding electron temperature $T_{\mathrm{e}}$ is shown in the same panels, and regional values of $T_{\mathrm{e}}$ are found in Table 3. (For reference, the line brightness profiles of [NII] and [OIII] are plotted in the respective panels so that the temperature variations can be correlated with the nebular surface brightness distribution.)
The surface brigthness in the [NII] lines is higher in the regions where the microstructures project, and this radiation may be safely attributed to them. From the middle left panel of Figs. 2 and 3 we see that the [NII] electron temperature is higher where the $\lambda 6583 \AA$ flux is lower, and $T_{\mathrm{e}}$ is lower where this flux is higher. Note that the [N II] lines used for deriving the temperature in the low-ionization gas were smoothed before forming the line ratio profiles, as noted in the previous section, so temperature fluctuations are suppressed. Pixel-bypixel values of $T_{\mathrm{e}}$ are shown in Figs. 2 and 3.

\subsubsection{FLIERs}

The temperature distribution of the FLIERs is difficult to determine with precision owing to noise. Nevertheless, some trends are apparent. Approaching from the left (star-facing) side of Fig. 2, $T_{\mathrm{e}}$ decreases from 11500 to about $10500 \mathrm{~K}$ at the peak. Subsequently approaching that starward peak of FLIER-b at $13.4 \mathrm{arcsec}, T_{\mathrm{e}}$ decreases to about $9800 \mathrm{~K}$ and then increases up to about $12000 \mathrm{~K}$, midway between the left and the right peaks of FLIER-b. At the right peak (14.4") $T_{\mathrm{e}}$ reaches a minimum of about $9400 \mathrm{~K}$ and then increases again to a maximum of $\approx 11000 \mathrm{~K}$, in correspondence with the minimum in the $\lambda 6583 \AA$ flux at $14.6^{\prime \prime}$. Afterwards the line flux decreases, but owing to noisy [N II] $\lambda 5755 \AA$ data, we cannot ascertain the detailed behaviour of the temperature. We feel we can claim to have measured in FLIER-b a [NII] temperature of $\approx 9800 \mathrm{~K}$ in the peak facing the central star and a lower $T_{\mathrm{e}}$ of $\approx 9400 \mathrm{~K}$ in the "outside" peak of FLIER-b.

The temperature between the two peaks of FLIER-b amounts to $\approx 12000 \mathrm{~K}$ close to the value outside the FLIER measured from [O III] lines of $\approx 11500 \mathrm{~K}$.

The anticorrelation between the behaviour of the temperature and of the line flux is easily interpreted in terms of an anticorrelation between the temperature and the density (see next subsection) although the electron density (from proper forbidden line ratios) is less precisely determined than the electron temperature.

\subsubsection{SLOWER}

The temperature in the SLOWER follows the same trend described for FLIER-b, with the same precise anticorrelation between the [NII] temperature and the $\lambda 6583 \AA$ flux across the whole microstructure, except (as it was for FLIER-b) in the zones where the [NII] line flux becomes quite low.

$T_{\mathrm{e}} \approx 9800 \mathrm{~K}$ in the peak facing the central star and $\approx 9600 \mathrm{~K}$ in the outside peak. In between it reaches $\approx 12000 \mathrm{~K}$.

\subsection{Ratio of lines of similar ionization potential}

It has been commonly done to compare the distribution of intensities of lines of very similar ionization potential in which ionization effects cancel out (to the first order). In this way it is possible to gain insight into abundance variations, temperature fluctuations, or very large densities that influence that emissivities of one line more than another. 
Plots of $R_{\mathrm{Ne}, \mathrm{O}}=[\mathrm{Ne}$ III] $] 33869 \AA /[\mathrm{O}$ III] $] 25007 \AA$ and $R_{\mathrm{N}, \mathrm{O}}=[\mathrm{N}$ II $] \lambda 6583 \AA /[\mathrm{O}$ II $] \lambda 3727 \AA$ are shown if Figs. 2 and 3. Both ratios show variations which correlate with the surface fluxes of the respective lines. We discuss each separately.

$R_{\mathrm{Ne}, \mathrm{O}}$ is sensitive to fluctuations in abundances, temperature (which appears to be constant; see Sect. 3), and ionization fraction of each species. There is no reason to expect fluctuations in $\mathrm{Ne} / \mathrm{O}$ since the central stars cannot synthesize either element. Both emission lines have very high critical densities, so density fluctuations cannot account for the variations in $R_{\mathrm{Ne}, \mathrm{O}}$. Charge transfer with some abundant ion, such as $\mathrm{H}$ or $\mathrm{He}$, is unlikely since the ionization fractions of both will be very close to unity throughout the $\mathrm{O}^{++}$and $\mathrm{Ne}^{++}$zones. So the only viable possibility is fluctuations in the ionization fractions of $\mathrm{O}^{++}$ and $\mathrm{Ne}^{++}$through the nebula. Perhaps at first sight this seems unlikely since these ions are created and destroyed at very similar ionization energies. However, a detailed model by AB97 showed that the ionization fraction of $\mathrm{Ne}^{++}, x\left(\mathrm{Ne}^{++}\right)$drops more slowly with distance from a central ionization source than does $x\left(\mathrm{O}^{++}\right)$, thus predicting that $R_{\mathrm{Ne}, \mathrm{O}}$ increases in the vicinity of an ionization front. The cause is the differing ionization potentials of $\mathrm{O}^{+}$and $\mathrm{Ne}^{+}$, from which the doubly ionized ions are formed by photoionization, and the hardening of stellar radiation. The predicted impact on $R_{\mathrm{Ne}, \mathrm{O}}$ matches the observations. These data represent the first direct confirmation of this prediction of which we are aware.

$R_{\mathrm{N}, \mathrm{O}}$ presents a different sort of situation from $R_{\mathrm{Ne}, \mathrm{O}}$. As we have already seen, $T_{\mathrm{e}}$ does fluctuate in the $\mathrm{O}^{+}$$\mathrm{N}^{+}$zone. Indeed, temperature fluctuations affect $R_{\mathrm{N}, \mathrm{O}}$ in proportion to $\exp \left(-16700 / T_{\mathrm{e}}\right)$. Charge transfer can be important too: AB97 showed that the effect of charge transfer near an ionization front - where the fraction of the electron donor $\mathrm{H}^{\circ}$ increases - can be dramatic. Ba94 asserted that N/O varies in FLIERs, though they did not observe NGC 7662 and they ignored charge transfer. Given the many possible physical influences on $R_{\mathrm{N}, \mathrm{O}}$, we are aware of the fact that any simple derivation of ionic abundances based on these lines must be regarded with caution.

The discussion thus far considers only photoionization and recombination process, but not mechanical heating by hydrodynamical processes. As we now describe, mechanical heating in and immediately around the FLIERs may be significant. Because the local densities are so high that radiative cooling dominates the energy budget, cloudlets that are hit by a hydrodynamical shock or by a radiation-driven shock will fragment quickly and easily (e.g., Mellema et al. 2002), due to the high compressibility of cooling gas, in association with local strong compression in the supersonic turbulence induced in the cloud.

Thus, the word "bullet" for these objects is a bad misnomer, and suggests altogether the wrong image. Rather, these "clouds" might be a swarm of smaller clouds, with substructures that are likely to continue all the way down to the local dissipation length scale. Consequently, the contact discontinuity between the external gas of the main nebula and the denser gas of the microstructure would have a very complex shape, and would be more properly thought of as a mixingand ablation layer that extends away from the swarm, in a direction determined by the local overall velocity gradient. The density and temperature structure of such a mixing layer is certainly capable of producing the observed changes in the $R_{\mathrm{N}, \mathrm{O}}$ and $R_{\mathrm{Ne}, \mathrm{O}}$ profiles.

The Leiden branch of the AstroHydro3D Project (Icke, Mellema, Rijkhorst \& Ritzerveld) is currently doing detailed work on this, which might clarify this interesting aspect. We note in passing that the "swarm" picture invoked here implies that our spectrograph slit averages over a large number of very small subcondensations (if, indeed, it is permitted to speak of "individual" condensations at all). Thus, line shapes and ratios are in fact complicated averages and, indeed, these averages are dependent on spatial resolution.

\subsection{Differences between FLIERs and SLOWERs}

In general the spectral behaviour of all line brightness profiles are very similar for the FLIERs and the SLOWER that we have considered in our spectroscopical analysis. Note, however, (cf. the upper-left panels of Figs. 2 and 3) that in the SLOWER the [OIII]5007 profile decreases significantly in the macro-structures ahead of the SLOWER and then increases strongly when approaching the SLOWER, while at the same time the [OII $] 3727$ profile increases even more rapidly towards the first "peak" of the SLOWER. In Fig. 2, on the contrary, the [OIII] profiles ahead of the FLIERs shows a plateau just before the brightest FLIERs (FLIER-b), and then its behaviour relative to [OII] 3727 closely follows that across and beyond the SLOWER in all details. It's not simple to understand the reasons for these differences.

With the present high spatial resolution observations, we do not see signatures of lines dominated by shock conditions as one would expect at the edges of the FLIERs, if the resolution is sufficient to separate the post-shock from its associated contact discontinuity. But, as we will see in the next sub-section, the actual thickness of this layer is likely much too small for shocks to be readily detectable. (We can rule out shocks as bright as those often found in $\mathrm{H}-\mathrm{H}$ objects, however.)

\subsection{Shocks?}

The thickness of the shock layers proper is of the order of the particle collision mean free path, which is much too small to resolve under the conditions observed in NGC 7662. The only layer which might in principle be resolved, is the compressed zone between the outermost (bow) shock and the contact discontinuity. In ordinary adiabatic flow, where the ratio of specific heats is $\gamma=5 / 3$, the thickness of such a layer is of the order of the physical size of the "bullet" that causes the bow shock. In the case of NGC 7662, that would be comparable to the transverse size of the FLIERs and SLOWERs. However, if the gas is strongly compressible due to cooling, the post-shock gas pressure that maintains the standoff distance between the shock and the "bullet" is undermined and the standoff layer collapses. Furthermore, the compressibility of the cooling gas causes any "bullet" to fragment, as stated above, so that the intrinsic length scales of the shock/contact layers become even smaller. Since the results of the detailed radiation 
hydrodynamics models are not available yet, we must refrain from stating whether or not the discontinuities can be resolved with HST.

It is worth noting that, given these complexities, it is highly remarkable that something managed to plow a path all the way to the periphery of the nebula, to form dense, rapidly cooling knots (that saves the subcondensation from early destruction by stellar photons) and yet to avoid the formation of shocks that can rip the knots apart (on time scales of the knot sound crossing time). Alternately, one might argue that the morphology of the FLIER through which our POS 1 slit crossed has a knot with a long sinewy tail. One can imagine that the sinewy tail is the photoablated or shock-evaporated material from the knot carried outward by ram or radiation pressure. Bear in mind that the knot and its tail have a high Doppler shift with respect to expansion speed of the surrounding medium (BPI). Therefore explanations of the origin of the knot and of the low ionization state of the tail remain unclear.

\subsection{Ionic abundances}

One should use a detailed photoionization code such as CLOUDY which includes a calculation of charge transfer in order to properly transform our line ratios into ionic and total abundances. But complications would then arise mainly due to the complex morphology of substructures and uncertainties in the prescription of the ionizating radiation field of the central star.

Moreover, if the FLIERs and SLOWERs are hydrodynamically interacting and heated, then the behaviour of strongly cooling subcondensations is hideously complex. A simple diagram showing the correlation between the gas density, the temperature, and the pressure in a realistic hydro computation of strongly compressible disk-wind interaction shows (Icke 2003) that the pressure scatters by a factor 100 and density over 1000; such an enormously inhomogeneous flow falls beyond the range of applicability of even a sophisticated code such as CLOUDY.

All in all we think it is reasonable to use a simplified procedure to gain information on the chemistry of nitrogen, where ground observations have suggested the mentioned enhancements, taking advantage of the quite higher spatial resolution HST offers to us.

We will use a simplified procedure similar to that used by Ba94, but improved to precisely take into account the effect of observational errors in the fluxes of individual emission lines and in the derived electron temperatures into deriving the ionic abundances, via a detailed error propagation across the whole procedure (Corradi et al. 1997; Perinotto \& Corradi 1998).

To this aim we have measured the observed flux of relevant emission lines in a number of positions across FLIER-a and FLIER-b in POS 1 and across the NE SLOWER in POS 2. These data are reported in Table 2. Uncertainties in the observed fluxes resulted to be dominated by STIS calibration errors, except in the faintest lines 5755 [NII] where the statistical error prevails. Total errors have been found to amount to $4-5 \%$ in all flux values reported in Table 2, except in the 5755 [NII] line, where the error was found to lie between 8 and $20 \%$ in the various locations. As a separate check, we see that the observed flux ratio of 6583 [NII] to 6548 [NII] confirms the above accuracy.

Resulting $\mathrm{Te}$ and the ionic abundance of NII/H by numbers are shown in Table 3. In parentheses are the percentage errors, calculated as above specified.

In the same table we also report values obtained by Barker (1986). He did use optical plus IUE observations in various positions within NGC 7662, termed by him Pos 1, 2, 3, 4, 5. Their offset locations in arcsec relative to the central star are $4 \mathrm{~N},(3.7 \mathrm{E}, 5 \mathrm{~S}), 8 \mathrm{~N}, 12 \mathrm{~N} \&(7.4 \mathrm{E}, 10 \mathrm{~S})$, respectively. Barker presents two sets of abundances for nitrogen: one using optical only lines, and another using UV + optical lines. Following his discussion we relay on his optical only results, considered to be quite reliable. These determinations have been obtained making use of the standard icf procedures and resulted in excellent agreement with results from the classical study in the same nebula with detailed photoionization model by Harrington et al. (1982).

We first examine the $\mathrm{N}+/ \mathrm{H}+$ abundance we have obtained in POS 1. Locations with central pixels " 145 " and " 151 " refer to the two peaks of FLIER-b. The fractional nitrogen abundance is of about $2 \times 10^{-5}$ in the two positions. We do not give weight to the fact that formal errors we obtain suggest that the two individual abundances are marginally different. The abundance is lower in location with central pixel "132", which is near to FLIER-a, but not precisely coincident with it.

Similar $T_{\mathrm{e}}$ and $\mathrm{N}+\mathrm{H}+$ abundances are found in POS 2, NE SLOWER: locations "150", "146", “139". A quite lower $\mathrm{N}+\mathrm{H}+$ is seen in location " 174 ", which refers to the rim macrostructure. Consistent with the much higher $T_{\mathrm{e}}(14600 \mathrm{~K})$ here measured.

We have also calculated the ionic abundances of $\mathrm{O}+/ \mathrm{H}+$ in POS 1 and POS 2 (not shown in Table 3). If we use the approximation $\mathrm{N}+/ \mathrm{O}+=\mathrm{N} / \mathrm{O}$, we obtain both in the FLIERs and in the SLOWER N/O $\approx 1 / 4$, just the value Barker obtains in the main nebular areas of NGC 7662 (see his Table 6). This on turn suggests that the nitrogen-to-hydrogen enhancement in FLIERs claimed by Ba94 in NGC 6543, 6826 and 7009 is not supported by the present HST observations in NGC 7662.

We are aware of the fact that the just mentioned approximation does not account for the charge-exchange reactions of oxygen and nitrogen with hydrogen. But also the results in the nebulae studied by Ba94 did not consider those reactions.

We have also found that there are no significant differences in the physical and chemical properties of FLIERs and SLOWERs in this nebula. This is consistent with the similar spectral behaviour of the two microstructures.

\section{Conclusions}

Observations with STIS on board the HST of NGC 7662 along two long-slit positons allowed us to clarify the physical conditions of ionization, temperature and density across the main body of the nebula and in the regions of microstrures called FLIERs (Fast Low Ionization Emission Regions) and SLOWERs (Slow Low Ionization Emission Regions), at a spatial resolution never achieved before. FLIERs 
Table 2. Observed line fluxes ${ }^{1}$.

\begin{tabular}{l|llll|llll}
\hline \hline & & \multicolumn{3}{|c|}{ POS 1 } & \multicolumn{4}{c}{ POS 2 } \\
\hline Pixel $^{2}$ & & $131-133$ & $144-146$ & $150-152$ & $173-175$ & $149-151$ & $145-147$ & $138-140$ \\
Arcsec $^{3}$ & & 10.8 & 13.4 & 14.6 & -7.8 & -12.5 & -13.3 & -15.0 \\
\hline$\lambda(\AA)$ & & & & & & & & \\
3727 & {$[\mathrm{OII}]$} & 474. & 783. & 1005. & 478. & 1990. & 1610. & 344. \\
4102 & $\mathrm{H} \delta$ & 205. & 153. & 156. & 144. & 276. & 211. & 68.0 \\
4341 & $\mathrm{H} \gamma$ & 347. & 260. & 245. & 214. & 467. & 365. & 117. \\
4861 & $\mathrm{H} \beta$ & 743. & 569. & 537. & 504. & 1040. & 783. & 254. \\
5755 & {$[\mathrm{NII}]$} & 6.10 & 9.6 & 14.4 & 6.9 & 28.0 & 22.1 & 3.0 \\
6300 & {$[\mathrm{OI}]$} & 29.0 & 83.5 & 167. & 14.0 & 248. & 232. & 5.6 \\
6548 & {$[\mathrm{NII}]$} & 90.0 & 174. & 281. & 58.4 & 476. & 415. & 58.0 \\
6563 & $\mathrm{H} \alpha$ & 2170. & 1690. & 1615. & 1210. & 2830. & 2340. & 710. \\
6583 & {$[\mathrm{NII}]$} & 302. & 564. & 885. & 201. & 1535. & 1330. & 187. \\
6717 & {$[\mathrm{SII}]$} & 20.4 & 32.8 & 53.6 & 13.2 & 86.8 & 107. & 17.7 \\
6731 & {$[\mathrm{SII}]$} & 28.2 & 48.8 & 68.2 & 20.0 & 149. & 166. & 23.0 \\
\hline
\end{tabular}

${ }^{1}$ In units of $10^{-15} \mathrm{erg} \mathrm{cm}^{-2} \mathrm{~s}^{-1}$.

2 See Fig. 1.

${ }^{3}$ Distance of central pixel from the star. POS 1: larger positives towards south; POS 2: larger negatives towards north-east.

Table 3. Chemical abundances.

\begin{tabular}{|c|c|c|c|c|c|c|c|c|c|}
\hline & \multicolumn{6}{|c|}{ POS 1} & & \multicolumn{2}{|c|}{ POS 2} \\
\hline Pixel $^{1}$ & $131-133$ & $144-146$ & & $150-152$ & & $173-175$ & $149-151$ & $145-147$ & $138-140$ \\
\hline $\operatorname{Arcsec}^{2}$ & 10.8 & 13.4 & & 14.6 & & -7.8 & -12.5 & -13.3 & -15.0 \\
\hline$T_{\mathrm{e}}(\mathrm{K})$ & $11000_{-600}^{+800}$ & $10200_{-500}^{+600}$ & & $10100_{-400}^{+500}$ & & $14600_{-1000}^{+1400}$ & $10500_{-300}^{+400}$ & $9900_{-400}^{+400}$ & $10100_{-300}^{+400}$ \\
\hline$N_{\mathrm{e}}\left(\mathrm{cm}^{-3}\right)$ & 1800 & 2300 & & 1400 & & 2500 & 4100 & 2700 & 1500 \\
\hline \multirow[t]{3}{*}{$\mathrm{NII} / \mathrm{H}$} & $4.8(-6)$ & $1.5(-5)$ & & $2.4(-5)$ & & $2.8(-6)$ & $2.2(-5)$ & $2.6(-5)$ & $1.2(-5)$ \\
\hline & (30) & (24) & & (18) & & (26) & (16) & (18) & (20) \\
\hline & \multicolumn{5}{|c|}{ Barker (1986) } & & & & \\
\hline Offset(arcsec) & $4 \mathrm{~N}$ & $3.7 \mathrm{E}, 5 \mathrm{~S}$ & $8 \mathrm{~N}$ & $12 \mathrm{~N}$ & 7.4E, $10 \mathrm{~S}$ & & & & \\
\hline Barker's pos & 1 & 2 & 3 & 4 & 5 & & & & \\
\hline$T_{\mathrm{e}}(\mathrm{K})$ & 13800 & 13100 & 12300 & 11600 & 11200 & & & & \\
\hline$N_{\mathrm{e}}\left(\mathrm{cm}^{-3}\right)$ & 4800 & 3200 & 4000 & 2400 & 5400 & & & & \\
\hline $\mathrm{NII} / \mathrm{H}$ & $2.1(-7)$ & $1.4(-7)$ & $2.5(-7)$ & $5.0(-7)$ & $3.7(-6)$ & & & & \\
\hline $\mathrm{N} / \mathrm{H}$ & $2.1:(-4)$ & $1.0(-4)$ & $6.9(-5)$ & $5.5(-5)$ & $2.3(-4)$ & & & & \\
\hline
\end{tabular}

${ }^{1}$ See Fig. 1.

${ }^{2}$ Distance of central pixel from the star. POS 1: larger positives towards south; POS 2: larger negatives towards north-east.

move supersonically relative to the ambient surrounding gases, while SLOWERs share the same expansion velocity of the ambient gases in which they are embedded. Because of this different velocity behaviour, FLIERs are expected to host shocks which should produce spectroscopical signatures, as enhancements e.g. in lines of the [SII] $6725 \AA$ doublet relative to the case of SLOWER.

Such enhancements are not seen. Indeed FLIERs do not show evidence of meaningful spectroscopic differences relative to SLOWERs.

Yet SLOWERs are simply interpreted as dense-lowionization knots ejected with the rest of the gas in the core of the PN whose high density allows it to recombine to lower ionization states as fast as stellar ultraviolet photoionization strips electrons.

Is the "thickness" of shocks in FLIERs too small to be resolved even with HST? If so, these shocks would be rather different from those observed in many $\mathrm{H}-\mathrm{H}$ objects, where the mentioned enhancements are easily seen also from ground observations.

Clearly signatures of enhancement in lines as [SII] $6725 \AA$ doublet are expected at the edges of FLIERs if the shocks were observable separately from the associated contact discontinuities. The state of art of hydrodynamical simulations does not 
yet allow us to predict the size of that thickness when we are not dealing with a simple object, but with a very inhomogenoeus configuration. We however argue that indeed these thicknesses are small due to strong cooling effects and probably below the spatial resolution even of HST. Progress in understanding the enigmatic nature of such nebular microstructures must wait for further advancements in the 3D hydrodynamical theory, which are under way in particular at Leiden University, and still better spatial resolution, possibly with the NGST.

Our observations show that the FLIERs and SLOWERs are characterized by densities of the order of $4000 \mathrm{~cm}^{-3}$ or perhaps more if low-density gas in the foreground and background pollutes our line measurements. This density is at least twice as high as the ambient nebular gas found in the region. We also find that the temperature of the $\mathrm{O}^{++}$zone is constant, but it refers to zones distinct from the microcondensations, while the $\mathrm{N}^{+}$temperature seems to decrease in the cores of the knots as if the local cooling rate is high where the emission measure peaks.

Unlike Ba94, we find spatial variations of the $[\mathrm{Ne} \mathrm{III}] /[\mathrm{O} \mathrm{III}]$ ratio, with the largest excursions near the cores of low-ionization structures. Following AB97 we attribute this to radiation hardening near ionization fronts which will increase the fraction of $\mathrm{Ne}^{++}$more than it does $\mathrm{O}^{++}$. Detailed numerical models are needed to understand these variations.

Finally we studied the problem of the enhancement of the abundance of $\mathrm{N}+/ \mathrm{H}+$ by factors of $2-5$ in the FLIERs found by $\mathrm{Ba} 94$ from ground observations in various PNe: NGC 6543, 6826, 7009.

In spite of problems still to be accounted for, such as a careful consideration of the role of charge-exchange reactions of nitrogen and oxygen with hydrogen, we find that in NGC 7662 our HST observations do not support the existence of enhancements in the nitrogen-to-hydrogen abundance in FLIERS.

Acknowledgements. We are grateful to Vincent Icke who helped with relevant comments and suggestions to improve a previous version of the paper. Support to one of the author (Bruce Balick) for program GO-8128 was provided by NASA through a grant from the Space Telescope Science Institute, which is operated by the Association of Universities for Research in Astronomy, Inc., under NASA contract NAS 5-26555. Support to Mario Perinotto and Patrizio Patriarchi was supplied by ASI (Italian Space Agency) through contract I/R/085/02.

\section{References}

Alexander, J., \& Balick, B. 1997, AJ, 114, 713 (AB97)

Balick, B. 1987, AJ, 94, 671

Balick, B., Preston, H. L., \& Icke, V. 1987, AJ, 94, 1641 (BPI)

Balick, B., Hajian, A. R., Terzian, Y., Perinotto, M., \& Patriarchi, P. 1998, AJ, 116, 360

Balick, B., Perinotto, M., Maccioni, A., Terzian, Y., \& Hajian, A. 1994, ApJ, 424, 800 (Ba94)

Barker, T. 1986, ApJ, 308, 314

Cardelli, J. A., Clayton, G. C., \& Mathis, J. S. 1989, ApJ, 345, 245

Corradi, R., Perinotto, M., Schwarz, H. E., \& Claeskens, J. F. 1997, A\&A, 322, 975

Dopita, M. 1997, ApJ, 485, L41

Frank, A., Balick, B., \& Livio, M. 1996, ApJ, 471, L53

Garcia-Segura, G. 1997, ApJ, 489, L189

Harrington, J. P., Seaton, M. J., Adams, S., \& Lutz, J. H. 1982, MNRAS, 199, 517

Harpaz, A., \& Soker, N. 1994, MNRAS, 270, 734

Hyung, S., \& Aller, L. H. 1997, ApJ, 491, 242

Icke, V. 2003, private communication

Livio, M. 1982, A\&A, 105, 37

Livio, M., \& Pringle, J. E. 1996, ApJ, 465, L55

Mellema, G. 1996, MNRAS, 277, 173

Mellema, G., Kurk, J., \& Röttgering, H. 2002, A\&A, 395, L13

Mellema, G., Raga, A. C., Canto, J., et al. 1998, A\&A, 331, 335

Osterbrock, D. E. 1989, Astrophysics of Gaseous Nebulae and Active Galactic Nuclei, University Science Books, 85

Pascoli, G. 1997, ApJ, 489, 946

Patriarchi, P., \& Perinotto, M. 1991, A\&AS, 91, 325

Perinotto, M. 2000, in Problems in Cosmic Gas Dynamics, Ap\&SS, 274,205

Perinotto, M., \& Corradi, R. 1998, A\&A, 332, 721

Redman, M. P., \& Dyson, J. E. 1999, MNRAS, 302, L17

Rubin, R. H., Bhatt, N. J., Dufour, R. J., et al. 2002, MNRAS, 334, 777

Soker, N. 1990, AJ, 99, 1869

Soker, N. 1992, ApJ, 389, 628

Soker, N., \& Livio, M. 1994, ApJ, 421, 219

Soker, N., \& Regev, O. 1998, AJ, 116, 2462

Steffen, W., Lopez, J. A., \& Lim, A. 2001, ApJ, 556, 823

Valdettaro, R., Patriarchi, P., \& Perinotto, M. 2001, in A decade of HST science, Space Telescope Science Institute Symp., 11-14 April 2000, Baltimore, MD, USA, ed. M. Livio, K. Noll, \& M. Stiavelli, 97 